\title{
A viscosity equation for minimizers of a class of very degenerate elliptic functionals
}

\author{
Giulio Ciraolo*
}

June 22, 2017

$$
\begin{aligned}
& \text { Abstract } \\
& \text { We consider the functional } \\
& \qquad J(v)=\int_{\Omega}[f(|\nabla v|)-v] d x,
\end{aligned}
$$

where $\Omega$ is a bounded domain and $f:[0,+\infty) \rightarrow \mathbb{R}$ is a convex function vanishing for $s \in[0, \sigma]$, with $\sigma>0$. We prove that a minimizer $u$ of $J$ satisfies an equation of the form

$$
\min \left(F\left(\nabla u, D^{2} u\right),|\nabla u|-\sigma\right)=0
$$

in the viscosity sense.

\section{Introduction}

Let $\Omega$ be a bounded domain in $\mathbb{R}^{N}, N \geq 2$, with boundary $\partial \Omega$ of class $C^{2, \alpha}$, with $0<\alpha<1$. We consider the variational problem

$$
\inf \left\{J(v): v \in W_{0}^{1, \infty}(\Omega)\right\}, \quad \text { where } \quad J(v)=\int_{\Omega}[f(|\nabla v|)-v] d x ;
$$

here, the function $f:[0,+\infty) \rightarrow \mathbb{R}$ is convex, monotone, nondecreasing and we assume that there exists $\sigma>0$ such that

$$
\begin{aligned}
& f \in C^{1}([0,+\infty)) \cap C^{3}((\sigma,+\infty)) \\
& f(0)=0 \text { and } \lim _{s \rightarrow+\infty} \frac{f(s)}{s}=+\infty \\
& f^{\prime}(s)=0 \text { for every } 0 \leq s \leq \sigma \\
& f^{\prime \prime}(s)>0 \text { for } s>\sigma
\end{aligned}
$$

Functionals of this kind occur in the study of complex-valued solutions of the eikonal equation (see [15]-[18]), as well as in the study of problems linked to traffic congestion (see [2]) and in variational problems which are relaxations of

\footnotetext{
*Dipartimento di Matematica e Informatica, Università di Palermo, Via Archirafi 34, 90123, Italy, (g.ciraolo@math.unipa.it).
} 
non-convex ones (see [5]). We have in mind the following two main examples of a function $f$ :

$$
f(s)= \begin{cases}0, & 0 \leq s \leq 1 \\ \frac{1}{2}\left[s \sqrt{s^{2}-1}-\log \left(s+\sqrt{s^{2}-1}\right)\right], & s>1\end{cases}
$$

which arises from the study of complex-valued solutions of the eikonal equation, and

$$
f(s)= \begin{cases}\frac{1}{q}(s-1)^{q}, & s>1, \\ 0, & 0 \leq s \leq 1,\end{cases}
$$

$q>1$, which is linked to traffic congestion problems.

Since $f$ vanishes in the interval $[0, \sigma]$, problem (11) is strongly degenerate and, as far as we know, few studies have been done. Besides the papers cited before, we mention [1] and [19] where regularity issues were tackled.

In this paper, we shall prove that the minimizer $u$ of (1) satisfies an equation of the form

$$
\min \left(F\left(\nabla u, D^{2} u\right),|\nabla u|-\sigma\right)=0
$$

in the viscosity sense (see Theorems 3.2 and 3.3 for the meaning of $F$ ).

Our strategy is to approximate $J$ by a sequence of less degenerating functionals so that the minimizers of the corresponding variational problems converge uniformly to $u$; this is done in Section 2 Then, the machinery of viscosity equations applies and, in Section 3 we prove that $u$ satisfies (5). To prove Theorems 3.2 and 3.3 which are our main results, we make use of techniques which have been used in the context of the $\infty$-Laplace operator (see for instance $[3,, 12,, 13])$.

\section{Preliminary results}

We start by recalling some well-known facts. Since $\Omega$ is bounded and $\partial \Omega$ is of class $C^{2, \alpha}$ then the following uniform exterior sphere condition holds: there exists $\rho>0$ such that for every $x_{0} \in \partial \Omega$ there exists a ball $B_{\rho}(y)$ of radius $\rho$ centered at $y=y\left(x_{0}\right) \in \mathbb{R}^{N} \backslash \bar{\Omega}$ such that $\overline{B_{\rho}(y)} \cap \bar{\Omega}=\overline{B_{\rho}(y)} \cap \partial \Omega$ and $x_{0} \in \partial B_{\rho}(y)$.

Notice that, since $f$ satisfies (2a) $-(2 \mathrm{~d})$, the functional $J$ is differentiable and a critical point $u$ of $J$ satisfies the problem

$$
\begin{cases}-\operatorname{div}\left(\frac{f^{\prime}(|\nabla u|)}{|\nabla u|} \nabla u\right)=1, & \text { in } \Omega \\ u=0, & \text { on } \partial \Omega\end{cases}
$$

in the weak sense, i.e.

$$
\int_{\Omega} \frac{f^{\prime}(|\nabla u|)}{|\nabla u|} \nabla u \cdot \nabla \phi d x=\int_{\Omega} \phi d x, \quad \text { for every } \phi \in C_{0}^{1}(\Omega) .
$$

It will be useful in the sequel to have at hand the solution of (6) when $\Omega$ is the ball of given radius $R$ (centered at the origin): it is given by

$$
u_{R}(x)=\int_{|x|}^{R} g^{\prime}\left(\frac{s}{N}\right) d s
$$


where

$$
g(t)=\sup \{s t-f(s): s \geq 0\}
$$

is the Fenchel conjugate of $f$ (see for instance [9] and [10]).

It is clear that, when $\sigma=0$, (11) has a unique solution, since $f$ is strictly convex. When $\sigma>0$, the uniqueness of a minimizer for (1) is proved in 7 .

In this section we shall approximate the functional $J$ by a sequence of strictly convex functionals

$$
J_{n}(v)=\int_{\Omega}\left[f_{n}(|\nabla v|)-v\right] d x,
$$

$n \in \mathbb{N}$, which are less degenerating than $J$ (see Proposition 2.3 for the assumptions on the functions $f_{n}$ ) and prove some uniform bounds for the minimizers $u_{n}$ of

$$
\inf \left\{J_{n}(v): v \in W_{0}^{1, \infty}(\Omega)\right\} .
$$

Notice that, if $f_{n} \in C^{1}([0,+\infty)) \cap C^{3}((0,+\infty))$ satisfies (2b) and it is such that $f_{n}^{\prime}(0)=0$ and $f_{n}^{\prime \prime}(s)>0$ for $s>0$, then the minimizer $u_{n}$ of (9) is unique and satisfies

$$
\int_{\Omega} \frac{f_{n}^{\prime}\left(\left|\nabla u_{n}\right|\right)}{\left|\nabla u_{n}\right|} \nabla u_{n} \cdot \nabla \phi d x=\int_{\Omega} \phi d x, \quad \text { for every } \phi \in C_{0}^{1}(\Omega) .
$$

We shall say that $w \in W^{1, \infty}(\Omega)$ is a subsolution of (11) if

$$
\int_{\Omega} \frac{f_{n}^{\prime}(|\nabla w|)}{|\nabla w|} \nabla w \cdot \nabla \phi d x \leq \int_{\Omega} \phi d x, \quad \text { for every } \phi \in C_{0}^{1}(\Omega) \text { with } \phi \geq 0,
$$

and that $w \in W^{1, \infty}(\Omega)$ is a supersolution of (11) if

$$
\int_{\Omega} \frac{f_{n}^{\prime}(|\nabla w|)}{|\nabla w|} \nabla w \cdot \nabla \phi d x \geq \int_{\Omega} \phi d x, \quad \text { for every } \phi \in C_{0}^{1}(\Omega) \text { with } \phi \geq 0 .
$$

Let $u_{n}$ and $v_{n}$ be a subsolution and a supersolutions of (11), respectively. Then, the following weak comparison principle holds: if $u_{n} \leq v_{n}$ on $\partial \Omega$ then $u_{n} \leq v_{n}$ in $\bar{\Omega}$ (see Lemma 3.7 in $[10$ ).

It will be useful to define the following $P$-function (see [10]):

$$
P_{n}(x)=\Phi_{n}\left(\left|\nabla u_{n}(x)\right|\right)+\frac{2}{N} u_{n}(x), \quad x \in \bar{\Omega},
$$

where

$$
\Phi_{n}(t)=2 \int_{0}^{t} s f_{n}^{\prime \prime}(s) d s .
$$

To avoid heavy notations, in Lemmas 2.1 and 2.2 we drop the dependence on $n$.

Lemma 2.1. Let $f \in C^{1}([0,+\infty)) \cap C^{3}((0,+\infty))$ be such that $f^{\prime}(0)=0$ and $f^{\prime \prime}(s)>0$ for $s>0$ and let $u$ be the solution of (10). Then, $|\nabla u|$ attains its maximum on the boundary of $\Omega$ and the following estimate holds:

$$
|\nabla u(x)| \leq M, \quad x \in \bar{\Omega},
$$


with

$$
M=g^{\prime}\left(\frac{\rho}{N-1}\left(e^{\frac{(N-1) R^{*}}{\rho}}-1\right)\right),
$$

where $g$ is the Fenchel conjugate of $f, R^{*}=\sup \{|x-y|: x, y \in \partial \Omega\}$ and $\rho$ is the radius of the uniform exterior sphere.

Furthermore,

$$
0 \leq u(x) \leq \min \left(\int_{0}^{R^{*}} g^{\prime}\left(\frac{s}{N}\right) d s, \frac{N}{2} \Phi(M)\right) \quad x \in \bar{\Omega} .
$$

Proof. Since $u$ is a minimizer of $J$, it is easy to show that $u \geq 0$. Being $R^{*}$ the diameter of $\Omega$, there exist a ball of radius $R^{*}$ that contains $\Omega$ (we can assume that such ball is centered at the origin). Since $u_{R^{*}}(x) \geq 0$ for $x \in \partial \Omega$, the weak comparison principle implies that

$$
u(x) \leq u_{R^{*}}(x) \text { for every } x \in \bar{\Omega} .
$$

From $u_{R^{*}}(x) \leq u_{R^{*}}(0), x \in B_{R^{*}}$ and from (8), we have

$$
u(x) \leq \int_{0}^{R^{*}} g^{\prime}\left(\frac{s}{N}\right) d s,
$$

for every $x \in \bar{\Omega}$.

Now, we consider the $P$-function given by (12). As proved in Lemma 3.2 in [10, $P$ attains its maximum on the boundary of $\Omega$ and thus

$$
P(x) \leq \max _{\partial \Omega} P=\max _{\partial \Omega} \Phi(|\nabla u|), \quad x \in \bar{\Omega} .
$$

Since $\Phi$ is strictly increasing, then we get

$$
\max _{\bar{\Omega}}|\nabla u(x)|=\max _{\partial \Omega}|\nabla u(x)|
$$

i.e. $|\nabla u|$ attains its maximum on the boundary of $\Omega$.

Following [11, we construct a barrier function for $u$ which will give us an upper bound for $|\nabla u|$ on the boundary of $\Omega$. Let $x_{0} \in \partial \Omega$ be fixed and let $B_{\rho}\left(y\left(x_{0}\right)\right)$ be the ball in the exterior sphere condition. Set

$$
\delta(x)=\operatorname{dist}\left(x, \partial B_{\rho}\left(y\left(x_{0}\right)\right)\right),
$$

and let $w(x)=\psi(\delta(x))$ be a function depending only on the distance from $\partial B_{\rho}\left(y\left(x_{0}\right)\right)$; we have

$$
\operatorname{div}\left\{f^{\prime}(|\nabla w|) \frac{\nabla w}{|\nabla w|}\right\}=\psi^{\prime \prime}(\delta(x)) f^{\prime \prime}\left(\psi^{\prime}(\delta(x))\right)+f^{\prime}\left(\psi^{\prime}(\delta(x))\right) \Delta \delta(x) .
$$

Since

$$
|\Delta \delta(x)|=\frac{N-1}{|x-y|} \leq \frac{N-1}{\rho},
$$


from (20) we obtain

$$
\operatorname{div}\left\{f^{\prime}(|\nabla w|) \frac{\nabla w}{|\nabla w|}\right\}+1 \leq \psi^{\prime \prime}(\delta(x)) f^{\prime \prime}\left(\psi^{\prime}(\delta(x))\right)+\frac{N-1}{\rho} f^{\prime}\left(\psi^{\prime}(\delta(x))\right)+1 .
$$

By choosing

$$
\psi(t)=\int_{0}^{t} g^{\prime}\left(\frac{\rho}{N-1}\left(e^{\frac{N-1}{\rho}\left(R^{*}-s\right)}-1\right)\right) d s,
$$

the right hand side of (21) vanishes and thus $w$ is a supersolution of (7). Notice that $\psi^{\prime}(t)>0$ for $t>0$ and then $\psi(t)>0$ for $t>0$. Since $x \in \Omega$ implies that $\operatorname{dist}\left(x, \partial B_{\rho}\left(x_{0}\right)\right)>0$, we have that $w(x) \geq 0$ for $x \in \bar{\Omega}$. The weak comparison principle yields $u(x) \leq w(x)$ in $\bar{\Omega}$. Since $x_{0} \in \partial \Omega$ is arbitrary, we obtain

$$
|\nabla u(x)| \leq g^{\prime}\left(\frac{\rho}{N-1}\left(e^{\frac{(N-1) R^{*}}{\rho}}-1\right)\right),
$$

for any $x \in \partial \Omega$. According to (19) the same estimate holds in the whole of $\Omega$ and (14) holds.

Notice that from (12)

$$
u(x) \leq \frac{N}{2} P(x), \quad x \in \bar{\Omega} ;
$$

since $P$ attains its maximum on the boundary of $\Omega$ and from (14) we have that

$$
u(x) \leq \frac{N}{2} \Phi(M)
$$

which, together with (18), implies (16).

We denote by $H_{\partial \Omega}(x)$ the mean curvature of $\partial \Omega$ at the point $x \in \partial \Omega$ and set

$$
H_{\partial \Omega}^{*}=\min _{x \in \partial \Omega} H_{\partial \Omega}(x) .
$$

In the following Lemma, we give a further bound for $u$ and $|\nabla u|$ in the case that the mean curvature of $\partial \Omega$ attains a positive minimum.

Lemma 2.2. Let $f$ be as in Lemma 2.1 and assume that $H_{\partial \Omega}^{*}>0$. Then,

$$
u(x) \leq \frac{N}{2} \Phi\left(g^{\prime}\left(\frac{1}{N H_{\partial \Omega}^{*}}\right)\right)
$$

and

$$
|\nabla u(x)| \leq g^{\prime}\left(\frac{1}{N H_{\partial \Omega}^{*}}\right)
$$

for every $x \in \bar{\Omega}$, where $\Phi$ is given by (13) and $g$ is the Fenchel conjugate of $f$.

Proof. Since $|\nabla u|>0$ on $\partial \Omega$ (see Lemma 2.7 in [7), equation (7) is nondegenerate in a neighborhood of $\partial \Omega$; from standard elliptic regularity theory (see [20] and [11), we know that $u \in C^{2, \alpha}(\bar{\Omega} \backslash\{x: \nabla u \neq 0\})$ for some $\alpha \in(0,1)$, and then (7) can be written pointwise on $\partial \Omega$ as

$$
f^{\prime \prime}\left(\left|u_{\nu}(x)\right|\right) u_{\nu \nu}(x)-(N-1) f^{\prime}\left(u_{\nu}(x)\right) H_{\partial \Omega}(x)=-1 ;
$$


here, $\nu$ denotes the exterior unit normal to $\partial \Omega, u_{\nu}=\nabla u \cdot \nu$ and $u_{\nu \nu}=\left(D^{2} u\right) \nu \cdot \nu$. From Lemma 3.3 in [10], we know that

$$
N f^{\prime}(|\nabla u(x)|) H_{\partial \Omega}(x) \leq 1,
$$

for every $x \in \partial \Omega$ and, since $g^{\prime}$ is nondecreasing, then

$$
|\nabla u(x)| \leq g^{\prime}\left(\frac{1}{N H_{\partial \Omega}^{*}}\right),
$$

for every $x \in \partial \Omega$. Since $\Phi$ is nondecreasing and $P$ (given by (12)) attains its maximum on $\partial \Omega$, from $u=0$ on $\partial \Omega$ we obtain

$$
P(x) \leq \Phi\left(g^{\prime}\left(\frac{1}{N H_{\partial \Omega}^{*}}\right)\right)
$$

for every $x \in \Omega$. From (12) and (24) we conclude.

Notice that, when $\Omega$ is a ball, (23) is optimal.

Proposition 2.3. Let $\left(f_{n}\right)_{n \in \mathbb{N}}$ be such that:

(i) $f_{n} \in C^{1}([0,+\infty)) \cap C^{3}((0,+\infty))$;

(ii) $f_{n}$ converges uniformly to $f$ on the compact sets contained in $[0 ;+\infty)$;

(iii) $f_{n}^{\prime}(0)=0$, the functions $f_{n}^{\prime}$ decrease to $f^{\prime}$ in $[0,+\infty)$ and $f_{n}^{\prime}$ converges uniformly to $f^{\prime}$ on the compact sets contained in $[0,+\infty)$

(iv) $f_{n}^{\prime \prime}(t)>0$ for $t>0$.

Let $u\left(\right.$ resp. $u_{n}$ ) be the solution of (11) for $J$ (resp. of (10) for $J_{n}$ ). Then

(a) $u_{n}$ is a minimizing sequence for $J$ and $J_{n}\left(u_{n}\right) \rightarrow J(u)$;

(b) $u_{n}$ and $\nabla u_{n}$ are uniformly bounded and (up to a subsequence) $\left(u_{n}\right)_{n \in \mathbb{N}}$ tends to $u$ in the sup norm topology and $u$ satisfies estimates (14) and (16) almost everywhere in $\Omega$.

Proof. Since $J_{n} \rightarrow J$ uniformly (a) is standard. Since the sequence $\left(f_{n}^{\prime}\right)_{n \in \mathbb{N}}$ is decreasing in $n$, then $g_{n}^{\prime}$ is increasing in $n$ and converges pointwise to $g^{\prime}$ (here, we denote by $g$ and $g_{n}$ the Fenchel conjugates of $f$ and $f_{n}$, respectively). Thus, $g_{n}(t) \leq g(t)$ and $g_{n}^{\prime}(t) \leq g^{\prime}(t)$ for every $t \in[0,+\infty)$ and (b) follows by Lemma 2.1 and an application of Ascoli-Arzelà's theorem.

\section{Viscosity Euler-Lagrange equation}

In this section we prove that the solution $u$ of (1) satisfies an equation of the form (5) in the viscosity sense. Firstly, we do it for $f \in C^{2}((0,+\infty)) \cup C^{3}((\sigma,+\infty))$ and then we deal with the case that $f$ is not twice differentiable at $s=\sigma$.

Consider a sequence of approximating functions $\left\{f_{n}\right\}_{n \in \mathbb{N}}$ satisfying $(i)-$ $-(i v)$ in Proposition 2.3. The minimizer $u_{n}$ for (10) satisfies

$$
-\operatorname{div} \frac{f_{n}^{\prime}\left(\left|\nabla u_{n}\right|\right)}{\left|\nabla u_{n}\right|}=1,
$$


in weak sense. Assume for a moment that $u_{n}$ is regular enough so that we can differentiate, then $u_{n}$ satisfies

$$
-\frac{\left|\nabla u_{n}\right| f_{n}^{\prime \prime}\left(\left|\nabla u_{n}\right|\right)-f_{n}^{\prime}\left(\left|\nabla u_{n}\right|\right)}{\left|\nabla u_{n}\right|^{3}} \Delta_{\infty} u_{n}-\frac{f_{n}^{\prime}\left(\left|\nabla u_{n}\right|\right)}{\left|\nabla u_{n}\right|} \Delta u_{n}=1,
$$

where

$$
\Delta_{\infty} u=\sum_{i, j=1}^{N} \frac{\partial^{2} u}{\partial x_{i} \partial x_{j}} \frac{\partial u}{\partial x_{i}} \frac{\partial u}{\partial x_{j}} .
$$

Since this equation is fully nonlinear and degenerate elliptic, it makes sense to define and study its viscosity solutions (see 4 ).

Let $P \in \mathbb{R}^{N}$ and $X \in \mathcal{S}^{N}$, where $\mathcal{S}^{N}$ is the space of real-valued $N \times N$ symmetric matrices. Consider the function

$$
F_{n}(P, X):= \begin{cases}-\frac{|P| f_{n}^{\prime \prime}(|P|)-f_{n}^{\prime}(|P|)}{|P|^{3}} P \cdot X P-\frac{f_{n}^{\prime}(|P|)}{|P|} \operatorname{tr}(X)-1, & P \neq 0, \\ -1, & P=0 .\end{cases}
$$

Notice that, if

$$
\lim _{s \rightarrow 0^{+}} \frac{s f_{n}^{\prime \prime}(s)-f_{n}^{\prime}(s)}{s^{3}}=0, \quad \text { and } \quad \lim _{s \rightarrow 0^{+}} \frac{f_{n}^{\prime}(s)}{s}=0,
$$

then $F_{n}$ is continuous. For future use, we shall assume that the sequence $\left\{f_{n}\right\}_{n \in \mathbb{N}}$ is such that

$$
\lim _{n \rightarrow+\infty} \frac{s f_{n}^{\prime \prime}(s)-f_{n}^{\prime}(s)}{s^{3}}=0 \quad \text { and } \quad \lim _{n \rightarrow+\infty} \frac{f_{n}^{\prime}(s)}{s}=0
$$

uniformly on the compact sets of $[0, \sigma)$; here, thanks to (26), the functions in the limits are understood as continuously extended to 0 at $s=0$.

We shall introduce the definition of viscosity solution of an equation of the form $F\left(\nabla v, D^{2} v\right)=0$ (see [13]).

Definition. An upper semicontinuous function $u$ defined in $\Omega$ is a viscosity subsolution of

$$
F\left(\nabla v, D^{2} v\right)=0,
$$

$x \in \Omega$, if, whenever $x_{0} \in \Omega$ and $\phi \in C^{2}(\Omega)$ are such that $u\left(x_{0}\right)=\phi\left(x_{0}\right)$ and $u(x)<\phi(x)$ if $x \neq x_{0}$, then

$$
F\left(\nabla \phi\left(x_{0}\right), D^{2} \phi\left(x_{0}\right)\right) \leq 0 .
$$

A lower semicontinuous function $u$ defined in $\Omega$ is a viscosity supersolution of (28) if, whenever $x_{0} \in \Omega$ and $\phi \in C^{2}(\Omega)$ are such that $u\left(x_{0}\right)=\phi\left(x_{0}\right)$ and $u(x)>\phi(x)$ if $x \neq x_{0}$, then

$$
F\left(\nabla \phi\left(x_{0}\right), D^{2} \phi\left(x_{0}\right)\right) \geq 0 .
$$

Finally, $u \in C^{0}(\Omega)$ is a viscosity solution of (28) if it is both a viscosity subsolution and a viscosity supersolution of (28). 
Lemma 3.1. Let $u_{n}$ be the minimizer of $J_{n}$, where $f_{n} \in C^{1}([0,+\infty)) \cup C^{3}((0,+\infty))$ satisfies (26) and is such that $f_{n}^{\prime \prime}(s)>0$ for $s>0$. Then $u_{n}$ is a viscosity solution of (28), with $F=F_{n}$ and $F_{n}$ given by (25).

Proof. Notice that, since $f_{n}$ satisfies (26), then $F_{n}$ is continuous. We present the details for the case of supersolutions. Let $x_{0} \in \Omega$ and $\phi \in C^{2}(\Omega)$ be such that $u_{n}\left(x_{0}\right)=\phi\left(x_{0}\right)$ and $u_{n}(x)>\phi(x)$ for $x \neq x_{0}$. Assume that $\nabla \phi\left(x_{0}\right) \neq 0$; we have to show that

$-\frac{\left|\nabla \phi\left(x_{0}\right)\right| f_{n}^{\prime \prime}\left(\left|\nabla \phi\left(x_{0}\right)\right|\right)-f_{n}^{\prime}\left(\left|\nabla \phi\left(x_{0}\right)\right|\right)}{\left|\nabla \phi\left(x_{0}\right)\right|^{3}} \Delta_{\infty} \phi\left(x_{0}\right)-\frac{f_{n}^{\prime}\left(\left|\nabla \phi\left(x_{0}\right)\right|\right)}{\left|\nabla \phi\left(x_{0}\right)\right|} \Delta \phi\left(x_{0}\right)-1 \geq 0$.

By contradiction, suppose that this is not the case. By continuity, there exists $r>0$ small enough such that

$$
-\frac{|\nabla \phi(x)| f_{n}^{\prime \prime}(|\nabla \phi(x)|)-f_{n}^{\prime}(|\nabla \phi(x)|)}{|\nabla \phi(x)|^{3}} \Delta_{\infty} \phi(x)-\frac{f_{n}^{\prime}(|\nabla \phi(x)|)}{|\nabla \phi(x)|} \Delta \phi(x)<1,
$$

for any $\left|x-x_{0}\right|<r$. Let $m=\inf \left\{u_{n}(x)-\phi(x):\left|x-x_{0}\right|=r\right\}$ and set $\eta=\phi+\frac{1}{2} m$. Since $m>0$ then $\eta<u_{n}$ on $\partial B_{r}\left(x_{0}\right), \eta\left(x_{0}\right)>u_{n}\left(x_{0}\right)$ and

$$
-\frac{|\nabla \eta(x)| f_{n}^{\prime \prime}(|\nabla \eta(x)|)-f_{n}^{\prime}(|\nabla \eta(x)|)}{|\nabla \eta(x)|^{3}} \Delta_{\infty} \eta(x)-\frac{f_{n}^{\prime}(|\nabla \eta(x)|)}{|\nabla \eta(x)|} \Delta \eta(x)<1,
$$

for any $\left|x-x_{0}\right|<r$. By multiplying by $\left(\eta-u_{n}\right)^{+}$, integrating in $B_{r}\left(x_{0}\right)$ and using an integration by parts, we have

$$
\int_{\left\{\eta>u_{n}\right\}} f_{n}^{\prime}(|\nabla \eta|) \frac{\nabla \eta}{|\nabla \eta|} \cdot \nabla\left(\eta-u_{n}\right) d x<\int_{\left\{\eta>u_{n}\right\}}\left(\eta-u_{n}\right) d x .
$$

Notice that, since $\eta\left(x_{0}\right)>u_{n}\left(x_{0}\right)$ and $\eta-u_{n}$ is continuous, the Lebesgue measure of $\left\{\eta>u_{n}\right\}$ is strictly positive. The function $\left(\eta-u_{n}\right)^{+}$extended to zero outside $B_{r}\left(x_{0}\right)$ can be used as a test function in (11):

$$
\int_{\left\{\eta>u_{n}\right\}} f_{n}^{\prime}\left(\left|\nabla u_{n}\right|\right) \frac{\nabla u_{n}}{\left|\nabla u_{n}\right|} \cdot \nabla\left(\eta-u_{n}\right) d x=\int_{\left\{\eta>u_{n}\right\}}\left(\eta-u_{n}\right) d x .
$$

Subtracting (30) from (29) we have

$$
\int_{\left\{\eta>u_{n}\right\}}\left[f_{n}^{\prime}(|\nabla \eta|) \frac{\nabla \eta}{|\nabla \eta|}-f_{n}^{\prime}\left(\left|\nabla u_{n}\right|\right) \frac{\nabla u_{n}}{\left|\nabla u_{n}\right|}\right] \cdot \nabla\left(\eta-u_{n}\right) d x<0 .
$$

Since

$$
\begin{aligned}
& {\left[f_{n}^{\prime}(|\nabla \eta|) \frac{\nabla \eta}{|\nabla \eta|}-f_{n}^{\prime}\left(\left|\nabla u_{n}\right|\right) \frac{\nabla u_{n}}{\left|\nabla u_{n}\right|}\right] \cdot \nabla\left(\eta-u_{n}\right)=} \\
& =f_{n}^{\prime}(|\nabla \eta|)|\nabla \eta|+f_{n}^{\prime}\left(\left|\nabla u_{n}\right|\right)\left|\nabla u_{n}\right|+ \\
& \quad-f_{n}^{\prime}(|\nabla \eta|) \frac{\nabla \eta}{|\nabla \eta|} \cdot \nabla u_{n}-f_{n}^{\prime}\left(\left|\nabla u_{n}\right|\right) \frac{\nabla u_{n}}{\left|\nabla u_{n}\right|} \cdot \nabla \eta
\end{aligned}
$$


Cauchy-Schwarz inequality yields

$$
\begin{aligned}
& {\left[f_{n}^{\prime}(|\nabla \eta|) \frac{\nabla \eta}{|\nabla \eta|}-f_{n}^{\prime}\left(\left|\nabla u_{n}\right|\right) \frac{\nabla u_{n}}{\left|\nabla u_{n}\right|}\right] \cdot \nabla\left(\eta-u_{n}\right) \geq} \\
& \quad \geq\left(f_{n}^{\prime}(|\nabla \eta|)-f_{n}^{\prime}\left(\left|\nabla u_{n}\right|\right)\right)\left(|\nabla \eta|-\left|\nabla u_{n}\right|\right)
\end{aligned}
$$

from the convexity of $f_{n}$ we obtain

$$
\left[f_{n}^{\prime}(|\nabla \eta|) \frac{\nabla \eta}{|\nabla \eta|}-f_{n}^{\prime}\left(\left|\nabla u_{n}\right|\right) \frac{\nabla u_{n}}{\left|\nabla u_{n}\right|}\right] \cdot \nabla\left(\eta-u_{n}\right) \geq 0,
$$

which gives the desired contradiction on account of (31).

To complete the proof that $u_{n}$ is a viscosity supersolution of (28), we shall prove that if $\phi$ is a test function touching $u_{n}$ at $x_{0}$ from below, then $\nabla \phi\left(x_{0}\right) \neq 0$ (i.e. the set of test functions touching $u_{n}$ from below with vanishing gradient is the empty set).

By contradition, let us assume that $\phi \in C^{2}(\Omega)$ is such that $u_{n}\left(x_{0}\right)=\phi\left(x_{0}\right)$, $u_{n}(x)>\phi(x)$ for $x \neq x_{0}$ and $\nabla \phi\left(x_{0}\right)=0$. Thus, there exists $c>0$ and $r_{1}>0$ such that $u_{n}(x)>\phi(x)>\psi(x)$ for $0<\left|x-x_{0}\right|<r_{1}$, where

$$
\psi(x)=-c\left|x-x_{0}\right|^{2}+u_{n}\left(x_{0}\right) .
$$

We notice that $\psi$ is of class $C^{2}$ and satisfies $F_{n}\left(\nabla \psi(x), D^{2} \psi(x)\right)<0$ for every $x$ in some ball of radius $r_{2}$ centered at $x_{0}$, i.e. there exists $r_{2}>0$ such that $\psi$ is a strict classical subsolution of $F_{n}\left(D v, D^{2} v\right)=0$ in $B_{r_{2}}\left(x_{0}\right)$.

Let $r=\min \left(r_{1}, r_{2}\right) / 2, m=\inf \left\{u_{n}(x)-\phi(x):\left|x-x_{0}\right|=r\right\}$ and set $\eta=$ $\phi+\frac{1}{2} m$. Notice that $\eta<u_{n}$ on $\partial B_{r}\left(x_{0}\right), \eta\left(x_{0}\right)>u_{n}\left(x_{0}\right)$ and $F_{n}\left(\nabla \eta, D^{2} \eta\right)<0$ in $B_{r}\left(x_{0}\right)$. As done in the first part of the proof, we use the function $\left(\eta-u_{n}\right)^{+}$ extended to zero outside $B_{r}\left(x_{0}\right)$ as a test function in (11) and we obtain (31); then, from (33) we get a contradiction. Thus, the set of test functions touching $u_{n}$ from below with vanishing gradient is the empty set and hence $u_{n}$ is a viscosity supersolution of (28).

To prove that $u_{n}$ is a subsolution of (28), we first consider a test function $\phi$ touching $u_{n}$ at $x_{0}$ from above with $\nabla \phi\left(x_{0}\right) \neq 0$. This case in analogous to the supersolution case. The case $\nabla \phi\left(x_{0}\right)=0$ is simpler than before, since in this case $F_{n}\left(\nabla \phi\left(x_{0}\right), D^{2} \phi\left(x_{0}\right)\right) \leq 0$ is straightforwardly satisfied.

Theorem 3.2. Let $u$ be the minimizer of (1), with $f$ satisfying (2) and $f \in$ $C^{2}((0,+\infty))$. Assume that there exists a sequence $\left\{f_{n}\right\}_{n \in \mathbb{N}}$ satisfying (i)-(iv) in Proposition 2.3. (26), (27) and such that $f_{n}^{\prime \prime}$ converges to $f^{\prime \prime}$ uniformly on the compact sets contained in $(0,+\infty)$.

Then, $u$ is a viscosity solution of

$$
\min \left(-\frac{|\nabla u| f^{\prime \prime}(|\nabla u|)-f^{\prime}(|\nabla u|)}{|\nabla u|^{3}} \Delta_{\infty} u-\frac{f^{\prime}(|\nabla u|)}{|\nabla u|} \Delta u-1,|\nabla u|-\sigma\right)=0 .
$$

Proof. Let $\left\{f_{n}\right\}_{n \in \mathbb{N}}$ be an approximating sequence of the function $f$ satisfying (i)-(iv) in Proposition 2.3, (26), (27) and such that $f_{n}^{\prime \prime}$ converges to $f^{\prime \prime}$ uniformly on the compact sets contained in $(0,+\infty)$. We refer to Theorem 3.5 for the existence of such a sequence in some relevant cases. From Proposition 2.3, we 
can assume that $u_{n}$ converges to $u$ uniformly as $n$ tends to infinity. By using a standard argument from the theory of viscosity solutions (see [8] and [14]), we shall prove that $u$ is a viscosity supersolution and subsolution of (34). The two proofs are not symmetric and we prove firstly that $u$ is a viscosity supersolution and then that it is also a viscosity subsolution.

Assume $\phi$ is a smooth function touching $u$ from below at $\hat{x} \in \Omega$, i.e., $u(\hat{x})=$ $\phi(\hat{x})$ and $u(x)>\phi(x)$ for any $x \neq \hat{x}$. Since $u_{n}$ is a viscosity solution of (28) and $u_{n}$ converges uniformly to $u$, there exist $\left\{x_{n}\right\}_{n \in \mathbb{N}} \subset \Omega$ such that

(i) for any $x \in \Omega, u_{n}\left(x_{n}\right)-\phi\left(x_{n}\right) \leq u_{n}(x)-\phi(x)$;

(ii) $x_{n}$ tends to $\hat{x}$ as $n$ tends to infinity;

see for instance [13] p. 95. Being $u_{n}$ a viscosity supersolution of (28), we can conclude that

$$
F_{n}\left(\nabla \phi\left(x_{n}\right), D^{2} \phi\left(x_{n}\right)\right) \geq 0 .
$$

Let assume that $|\nabla \phi(\hat{x})|<\sigma$; since $\phi$ is of class $C^{2}$ and from (ii), there exists $\delta>0$ such that $\left|\nabla \phi\left(x_{n}\right)\right| \leq \sigma-\delta$ for $n$ large enough. By taking the limit as $n \rightarrow \infty$ and from (27) we get a contradiction. Thus, we may exclude that $|\nabla \phi(\hat{x})|<\sigma$.

Now assume that $|\nabla \phi(\hat{x})| \geq \sigma$. Since $f_{n}^{\prime}$ and $f_{n}^{\prime \prime}$ converge uniformly on compact sets to $f^{\prime}$ and $f^{\prime \prime}$, respectively, by taking the limit as $n \rightarrow \infty$ we get that both

$$
-\frac{|\nabla \phi(\hat{x})| f^{\prime \prime}(|\nabla \phi(\hat{x})|)-f^{\prime}(|\nabla \phi(\hat{x})|)}{|\nabla \phi(\hat{x})|^{3}} \Delta_{\infty} \phi(\hat{x})-\frac{f^{\prime}(|\nabla \phi(\hat{x})|)}{|\nabla \phi(\hat{x})|} \Delta \phi(\hat{x})-1 \geq 0,
$$

and

$$
|\nabla \phi(\hat{x})|-\sigma \geq 0
$$

are satisfied. Hence the claim is proven.

Now, we prove that $u$ is a viscosity subsolution of (34). Assume $\phi$ is a smooth function such that $u(\hat{x})=\phi(\hat{x})$ and $u(x)<\phi(x)$ for any $x \neq \hat{x}$. As claimed at the previous case, there exists a sequence $\left\{x_{n}\right\}_{n \in \mathbb{N}}$ such that

(i) $u_{n}\left(x_{n}\right)-\phi\left(x_{n}\right) \geq u_{n}(x)-\phi(x)$;

(ii) $x_{n}$ tends to $\hat{x}$ as $n$ tends to infinity.

If $|\nabla \phi(\hat{x})| \leq \sigma$, then obviously

$$
\begin{array}{r}
\min \left(-\frac{|\nabla \phi(\hat{x})| f^{\prime \prime}(|\nabla \phi(\hat{x})|)-f^{\prime}(|\nabla \phi(\hat{x})|)}{|\nabla \phi(\hat{x})|^{3}} \Delta_{\infty} \phi(\hat{x})-\frac{f^{\prime}(|\nabla \phi(\hat{x})|)}{|\nabla \phi(\hat{x})|} \Delta \phi(\hat{x})-1\right. \\
|\nabla \phi(\hat{x})|-\sigma) \leq 0
\end{array}
$$

holds. In case $|\nabla \phi(\hat{x})|>\sigma$, then $\left|\nabla \phi\left(x_{n}\right)\right| \geq \sigma+\delta$ for some $\delta>0$ and for any $n$ large enough. Since $u_{n}$ is a viscosity subsolution of (28), then we have $F_{n}\left(\nabla \phi\left(x_{n}\right), D^{2} \phi\left(x_{n}\right)\right) \leq 0$. Since $f_{n}$ and its first and second derivatives converges uniformly as $n \rightarrow+\infty$, by taking the limit leads to

$$
-\frac{|\nabla \phi(\hat{x})| f^{\prime \prime}(|\nabla \phi(\hat{x})|)-f^{\prime}(|\nabla \phi(\hat{x})|)}{|\nabla \phi(\hat{x})|^{3}} \Delta_{\infty} \phi(\hat{x})-\frac{f^{\prime}(|\nabla \phi(\hat{x})|)}{|\nabla \phi(\hat{x})|} \Delta \phi(\hat{x})-1 \leq 0,
$$


which completes the proof.

Now, we assume that $f$ satisfies (2) and

$$
\lim _{s \rightarrow \sigma^{+}} f^{\prime \prime}(s)=+\infty .
$$

Thus, $f \notin C^{2}((0,+\infty))$ (i.e. $f$ is not twice differentiable at $\left.s=\sigma\right)$. Since it is not possible to choose $f_{n}$ such that $f_{n}^{\prime \prime}$ converges uniformly to $f^{\prime \prime}$, we can not proceed as in Theorem 3.2

Let

$$
a(s)= \begin{cases}\frac{f^{\prime}(s)}{s f^{\prime \prime}(s)}, & s>\sigma, \\ 0, & 0 \leq s \leq \sigma,\end{cases}
$$

and

$$
b(s)= \begin{cases}\frac{s^{2}}{f^{\prime \prime}(s)}, & s>\sigma, \\ 0, & 0 \leq s \leq \sigma .\end{cases}
$$

Notice that $a, b \in C^{0}([0,+\infty))$. Analogously, we define

$$
a_{n}(s)=\frac{f_{n}^{\prime}(s)}{s f_{n}^{\prime \prime}(s)} \text { and } b_{n}(s)=\frac{s^{2}}{f_{n}^{\prime \prime}(s)},
$$

for $s>0$.

Theorem 3.3. Let $u$ be the minimizer of (11), with $f$ satisfying (21) and (35). Assume that there exists a sequence $\left\{f_{n}\right\}_{n \in \mathbb{N}}$ satisfying (i)-(iv) in Proposition 2.3. (26) and (27). Let $a, b, a_{n}, b_{n}$ be defined by (36) -(38) and assume that $f_{n}$ is such that $a_{n}$ and $b_{n}$ converge uniformly to $a$ and $b$ in the compact sets contained in $(0,+\infty)$ and $(\sigma,+\infty)$, respectively.

Then $u$ is a viscosity solution of

$$
\min \left(-[1-a(|\nabla u|)] \Delta_{\infty} u-|\nabla u|^{2} a(|\nabla u|) \Delta u-b(|\nabla u|),|\nabla u(x)|-\sigma\right)=0 .
$$

Proof. The proof splits in two parts. First we prove that $u$ is a viscosity supersolution, then that it is also a subsolution. The earlier is slightly more involved and we deal with it first. Notice that the existence of the sequence $\left\{f_{n}\right\}_{n \in \mathbb{N}}$ is proved in Theorem 3.5 for some relevant cases.

The function $u$ is a viscosity supersolution of (39).

Assume $\phi$ is a smooth function touching $u$ from below at $\hat{x} \in \Omega$, i.e., $u(\hat{x})=\phi(\hat{x})$ and $u(x)>\phi(x)$ for any $x \neq \hat{x}$. Recall that $u_{n}$ is a viscosity solution of (28) and that, from Proposition 2.3. we can assume that $u_{n}$ converges uniformly to $u$ as $n$ tends to infinity. Thus, there exist $\left\{x_{n}\right\}_{n \in \mathbb{N}} \subset \Omega$ such that for any $x \in \Omega$, $u_{n}\left(x_{n}\right)-\phi\left(x_{n}\right) \leq u_{n}(x)-\phi(x)$ and $x_{n}$ tends to $\hat{x}$ as $n$ tends to infinity.

Since $u_{n}$ is a viscosity supersolution of (28), we can conclude that

$$
F_{n}\left(\nabla \phi\left(x_{n}\right), D^{2} \phi\left(x_{n}\right)\right) \geq 0 .
$$

Let assume that $|\nabla \phi(\hat{x})|<\sigma$. As done in the proof of Theorem 3.2, we get a contradiction and we may exclude that $|\nabla \phi(\hat{x})|<\sigma$. 
Now assume that $|\nabla \phi(\hat{x})|>\sigma$. Hence, we may assume that $\left|\nabla \phi\left(x_{n}\right)\right|>\sigma$ (at least for $n$ large). By multiplying both sides of (40) by

$$
\frac{\left|\nabla \phi\left(x_{n}\right)\right|^{2}}{f_{n}^{\prime \prime}\left(\left|\nabla \phi\left(x_{n}\right)\right|\right)}
$$

we have

$-\left[1-a_{n}\left(\left|\nabla \phi\left(x_{n}\right)\right|\right)\right] \Delta_{\infty} \phi\left(x_{n}\right)-\left|\nabla \phi\left(x_{n}\right)\right|^{2} a_{n}\left(\left|\nabla \phi\left(x_{n}\right)\right|\right) \Delta \phi\left(x_{n}\right)-b_{n}\left(\left|\nabla \phi\left(x_{n}\right)\right|\right) \geq 0$.

From the uniform convergence of $a_{n}$ and $b_{n}$ and by taking the limit as $n \rightarrow \infty$, we get that both

$$
-[1-a(|\nabla \phi(\hat{x})|)] \Delta_{\infty} \phi(\hat{x})-|\nabla \phi(\hat{x})|^{2} a(|\nabla \phi(\hat{x})|) \Delta \phi(\hat{x})-b(|\nabla \phi(\hat{x})|) \geq 0,
$$

and

$$
|\nabla \phi(\hat{x})|-\sigma \geq 0
$$

are satisfied.

It remains to consider the case $|\nabla \phi(\hat{x})|=\sigma$. Since we do not have the uniform convergence of $b_{n}$ to $b$ in a neighborhood of $\sigma$, we must proceed in a different way. By contradiction, let us assume that $u$ is not a viscosity supersolution of (39). For what we have proven in the first part of the proof, there exist $\hat{x} \in \Omega$ and a smooth function $\phi$ touching $u$ from below at $\hat{x} \in \Omega$ with $|\nabla \phi(\hat{x})|=\sigma$ such that

$$
\begin{array}{r}
\min \left(-[1-a(|\nabla \phi(\hat{x})|)] \Delta_{\infty} \phi(\hat{x})-|\nabla \phi(\hat{x})|^{2} a(|\nabla \phi(\hat{x})|) \Delta \phi(\hat{x})-b(|\nabla \phi(\hat{x})|)\right. \\
|\nabla \phi(\hat{x})|-\sigma)<0
\end{array}
$$

Since $|\nabla \phi(\hat{x})|=\sigma$, then $a(\sigma)=b(\sigma)=0$ and the above inequality yields

$$
-\Delta_{\infty} \phi(\hat{x})<0
$$

As done before, there exists $\left\{x_{n}\right\}_{n \in \mathbb{N}} \subset \Omega$ such that for any $x \in \Omega, u_{n}\left(x_{n}\right)-$ $\phi\left(x_{n}\right) \leq u_{n}(x)-\phi(x)$ and $x_{n}$ tends to $\hat{x}$ as $n$ tends to infinity. Notice that $u_{n}$ is a viscosity supersolution of (28) and then

$$
-\frac{f_{n}^{\prime \prime}\left(\left|\nabla \phi\left(x_{n}\right)\right|\right)}{\left|\nabla \phi\left(x_{n}\right)\right|^{2}} \Delta_{\infty} \phi\left(x_{n}\right) \geq 1+\frac{f_{n}^{\prime}\left(\left|\nabla \phi\left(x_{n}\right)\right|\right)}{\left|\nabla \phi\left(x_{n}\right)\right|} \Delta \phi\left(x_{n}\right)-\frac{f_{n}^{\prime}\left(\left|\nabla \phi\left(x_{n}\right)\right|\right)}{\left|\nabla \phi\left(x_{n}\right)\right|^{3}} \Delta_{\infty} \phi\left(x_{n}\right) .
$$

Since $x_{n}$ converges to $\hat{x}$ and $\phi$ is of class $C^{2}$, then $\left|\nabla \phi\left(x_{n}\right)\right|$ converges to $\sigma$ and, from (41), $\Delta_{\infty} \phi\left(x_{n}\right)>0$ for $n$ large enough. The uniform convergence of $f_{n}^{\prime}$ to $f^{\prime}$ yields the following contradiction:

$$
\frac{1}{2} \leq-\frac{f^{\prime \prime}\left(\left|\nabla \phi\left(x_{n}\right)\right|\right)}{\left|\nabla \phi\left(x_{n}\right)\right|^{2}} \Delta_{\infty} \phi\left(x_{n}\right)<0
$$

for $n$ large enough. Hence the claim is proven.

The function $u$ is a viscosity subsolution of (39).

Assume $\phi$ is a smooth function such that $u(\hat{x})=\phi(\hat{x})$ and $u(x)<\phi(x)$ for any $x \neq \hat{x}$. Thus, there exists a sequence $\left\{x_{n}\right\}_{n \in \mathbb{N}}$ such that $u_{n}\left(x_{n}\right)-\phi\left(x_{n}\right) \geq$ $u_{n}(x)-\phi(x)$ and $x_{n}$ tends to $\hat{x}$ as $n$ tends to infinity. 
If $|\nabla \phi(\hat{x})| \leq \sigma$, then obviously

$$
\begin{array}{r}
\min \left(-[1-a(|\nabla \phi(\hat{x})|)] \Delta_{\infty} \phi(\hat{x})-|\nabla \phi(\hat{x})|^{2} a(|\nabla \phi(\hat{x})|) \Delta \phi(\hat{x})-b(|\nabla \phi(\hat{x})|),\right. \\
|\nabla \phi(\hat{x})|-\sigma) \leq 0
\end{array}
$$

holds. In case $|\nabla \phi(\hat{x})|>\sigma$, from the fact that $u_{n}$ is a viscosity subsolution of (28), we can conclude (carrying out the same algebraic manipulation showed at the previous step)

$-\left[1-a_{n}\left(\left|\nabla \phi\left(x_{n}\right)\right|\right)\right] \Delta_{\infty} \phi\left(x_{n}\right)-\left|\nabla \phi\left(x_{n}\right)\right|^{2} a_{n}\left(\left|\nabla \phi\left(x_{n}\right)\right|\right) \Delta \phi\left(x_{n}\right)-b_{n}\left(\left|\nabla \phi\left(x_{n}\right)\right|\right) \leq 0$.

Taking the limit leads to the desired conclusion.

Remark 3.4. It is of interest to have an analogue of Theorem 3.3 when $f$ satisfies

$$
0<\lim _{s \rightarrow \sigma^{+}} f^{\prime \prime}(s)<+\infty .
$$

This case can be studied by using an argument analogue to the one used in the proof of Theorem 3.3 and under the additional assumption

$$
\left(\limsup _{n \rightarrow \infty} f_{n}^{\prime \prime}\right)(\sigma) \leq \lim _{s \rightarrow \sigma^{+}} f^{\prime \prime}(s) .
$$

We will not write in this paper the details of the proof. We just mention that if $f$ is given by (4) with $q=2$, then the approximating sequence given in the proof of the following Theorem satisfies this additional assumption.

Theorems 3.2 and 3.3 require the existence of a sequence $\left\{f_{n}\right\}_{n \in \mathbb{N}}$ which satisfies several assumptions. In the following theorem, we construct an explicit example.

Theorem 3.5. Let $f$ satisfy (21) and let a be given by (36). Assume that there exists $\tilde{\sigma}>\sigma$ such that $a(s)$ is nondecreasing for $s \in[\sigma, \tilde{\sigma}]$. Then there exists $\left\{f_{n}\right\}_{n \in \mathbb{N}}$ which satisfies the assumptions required in Theorems 3.2 and 3.3 .

Proof. We construct an explicit example. A convenient way to construct the sequence $\left\{f_{n}\right\}_{n \in \mathbb{N}}$ is to modify $f^{\prime}$ only in the interval $(0, \sigma+\varepsilon)$, with $\varepsilon>0$ small enough. We define

$$
f_{\varepsilon}^{\prime}(s)= \begin{cases}f^{\prime}(\sigma+\varepsilon)\left[2\left(\frac{s}{\sigma+\varepsilon}\right)^{p_{\varepsilon}}-\left(\frac{s}{\sigma+\varepsilon}\right)^{q_{\varepsilon}}\right], & 0 \leq s \leq \sigma+\varepsilon \\ f^{\prime}(s), & s>\sigma+\varepsilon\end{cases}
$$

with

$$
p_{\varepsilon}=\frac{(\sigma+\varepsilon) f^{\prime \prime}(\sigma+\varepsilon)}{f^{\prime}(\sigma+\varepsilon)}\left(1+\frac{1}{2} \omega_{\varepsilon}\right)
$$

and

$$
q_{\varepsilon}=\frac{(\sigma+\varepsilon) f^{\prime \prime}(\sigma+\varepsilon)}{f^{\prime}(\sigma+\varepsilon)}\left(1+\omega_{\varepsilon}\right)
$$

where,

$$
\omega_{\varepsilon}=\sqrt{2\left[1-\frac{f^{\prime \prime \prime}(\sigma+\varepsilon) f^{\prime}(\sigma+\varepsilon)}{f^{\prime \prime}(\sigma+\varepsilon)^{2}}-\frac{f^{\prime}(\sigma+\varepsilon)}{(\sigma+\varepsilon) f^{\prime \prime}(\sigma+\varepsilon)}\right]} .
$$


Since $a(s)$ is nondecreasing in $[\sigma, \tilde{\sigma}]$, then the same holds for the $\log a(s)$. Thus,

$$
0 \leq \frac{d}{d s} \log a(s)=\frac{f^{\prime \prime}(s)}{f^{\prime}(s)}-\frac{1}{s}-\frac{f^{\prime \prime \prime}(s)}{f^{\prime \prime}(s)} .
$$

By multiplying by $f^{\prime}(s) / f^{\prime \prime}(s)$ we get

$$
\frac{f^{\prime}(s)}{s f^{\prime \prime}(s)}+\frac{f^{\prime}(s) f^{\prime \prime \prime}(s)}{f^{\prime \prime}(s)^{2}} \leq 1
$$

for $s \in(\sigma, \tilde{\sigma})$, which implies that $\omega_{\varepsilon}$ is well-defined.

Tedious but straightforward computations show that $f_{\varepsilon} \in C^{3}(0,+\infty) \cap$ $C^{1}([0,+\infty))$. Since we modified $f^{\prime}$ only on a compact set, it is easy to show the uniform convergence of $f_{n}$ and $f_{n}^{\prime}$ to $f$ and $f^{\prime}$, respectively.

Notice that

$$
f_{\varepsilon}^{\prime \prime}(s)= \begin{cases}\frac{f^{\prime}(\sigma+\varepsilon)}{\sigma+\varepsilon}\left[2 p_{\varepsilon}\left(\frac{s}{\sigma+\varepsilon}\right)^{p_{\varepsilon}-1}-q_{\varepsilon}\left(\frac{s}{\sigma+\varepsilon}\right)^{q_{\varepsilon}-1}\right], & 0 \leq s \leq \sigma+\varepsilon \\ f^{\prime \prime}(s), & s>\sigma+\varepsilon\end{cases}
$$

since $2 p_{\varepsilon}>q_{\varepsilon}$ and $p_{\varepsilon}<q_{\varepsilon}$, then $f_{\varepsilon}^{\prime \prime}>0$.

Notice that we have

$$
\lim _{s \rightarrow \sigma^{+}} a(s)=0 .
$$

Indeed, assume by contradiction that there exists $\alpha>0$ such that $a(s) \rightarrow 1 / \alpha$ as $s \rightarrow \sigma^{+}$. Since $a(s)$ is nondecreasing, then $a(s) \geq 1 / \alpha$ for any $s \in[\sigma, \tilde{\sigma}]$, which implies that

$$
\frac{d}{d s} \log f^{\prime}(s) \leq \frac{d}{d s} \log s^{\alpha}, \quad s \in(\sigma, \tilde{\sigma})
$$

By integrating both sides of the above inequality from $s$ to $\tilde{\sigma}$ and after simple manipulations, we obtain that

$$
f^{\prime}(s) \geq f^{\prime}(\tilde{\sigma})\left(\frac{s}{\tilde{\sigma}}\right)^{\alpha}
$$

for any $s \in(\sigma, \tilde{\sigma})$. By taking the limit as $s \rightarrow \sigma^{+}$, we obtain $f^{\prime}(\sigma)>0$, a contradiction. Thus, (43) holds.

Since $p_{\varepsilon}, q_{\varepsilon} \geq(\sigma+\varepsilon) f^{\prime \prime}(\sigma+\varepsilon) / f^{\prime}(\sigma+\varepsilon)$, from (43) we have

$$
\lim _{\varepsilon \rightarrow 0^{+}} p_{\varepsilon}=+\infty \text { and } \lim _{\varepsilon \rightarrow 0^{+}} q_{\varepsilon}=+\infty
$$

Assume that $\varepsilon$ is small enough so that $p_{\varepsilon}$ and $q_{\varepsilon}$ are greater than 3 ; then

$\frac{s f_{\varepsilon}^{\prime \prime}(s)-f_{\varepsilon}^{\prime}(s)}{s^{3}}=f^{\prime}(\sigma+\varepsilon)\left[\frac{2\left(p_{\varepsilon}-1\right)}{(\sigma+\varepsilon)^{p_{\varepsilon}}} s^{p_{\varepsilon}-3}-\frac{\left(q_{\varepsilon}-1\right)}{(\sigma+\varepsilon)^{q_{\varepsilon}}} s^{q_{\varepsilon}-3}\right], \quad 0<s \leq \sigma+\varepsilon$,

and

$$
\frac{f_{\varepsilon}^{\prime}(s)}{s}=f^{\prime}(\sigma+\varepsilon)\left[\frac{2 p_{\varepsilon}}{(\sigma+\varepsilon)^{p_{\varepsilon}}} s^{p_{\varepsilon}-1}-\frac{q_{\varepsilon}}{(\sigma+\varepsilon)^{q_{\varepsilon}}} s^{q_{\varepsilon}-1}\right], \quad 0<s \leq \sigma+\varepsilon .
$$

From (44), we get (26) and (27). 
We notice that

$$
a_{\varepsilon}(s)=\frac{f^{\prime}(\sigma+\varepsilon)}{(\sigma+\varepsilon) f^{\prime \prime}(\sigma+\varepsilon)} \cdot \frac{2\left(\frac{s}{\sigma+\varepsilon}\right)^{p_{\varepsilon}}-\left(\frac{s}{\sigma+\varepsilon}\right)^{q_{\varepsilon}}}{2\left(\frac{s}{\sigma+\varepsilon}\right)^{p_{\varepsilon}}-\left(\frac{s}{\sigma+\varepsilon}\right)^{q_{\varepsilon}}+\omega_{\varepsilon}\left[\left(\frac{s}{\sigma+\varepsilon}\right)^{p_{\varepsilon}}-\left(\frac{s}{\sigma+\varepsilon}\right)^{q_{\varepsilon}}\right]},
$$

for $0 \leq s \leq \sigma+\varepsilon$ and $a_{\varepsilon}(s)=a(s)$ for $s>\sigma+\varepsilon$. Thus,

$$
\sup _{s \in \mathbb{R}}\left|a_{\varepsilon}(s)-a(s)\right| \leq \frac{f^{\prime}(\sigma+\varepsilon)}{(\sigma+\varepsilon) f^{\prime \prime}(\sigma+\varepsilon)}
$$

by (43), we obtain that $a_{\varepsilon}$ converges uniformly to $a$.

Since $f_{\varepsilon}^{\prime \prime}(s)=f^{\prime \prime}(s)$ for $s \geq \sigma+\varepsilon$, it is clear that $b_{n}$ converges uniformly to $b$ in the compact sets contained in $(\sigma,+\infty)$.

Remark 3.6. We notice when $f$ is given by (3) or (4), then a satisfies the assumptions of Theorem 3.5. Indeed, if $f$ is given by (3), then we have

$$
a(s)= \begin{cases}0, & 0 \leq s \leq 1 \\ 1-\frac{1}{s^{2}}, & s>1\end{cases}
$$

and then $a(s)$ is nondecreasing.

When $f$ is given by (4), then

$$
a(s)= \begin{cases}0, & 0 \leq s \leq 1, \\ \frac{1}{q-1}\left(1-\frac{1}{s}\right), & s>1\end{cases}
$$

which is a nondecreasing function.

Example 3.7. Let $f$ be given by (3). Then

$$
f^{\prime}(s)=\sqrt{\left(s^{2}-1\right)^{+}}
$$

and $a$ and $b$ in (36) and (37) read as

$$
a(s)= \begin{cases}0, & 0 \leq s \leq 1 \\ 1-\frac{1}{s^{2}}, & s>1\end{cases}
$$

and

$$
b(s)=s \sqrt{\left(s^{2}-1\right)^{+}} .
$$

We notice that, working as in the proof of Theorem 3.3 , we can prove that $u$ satisfies other equations in the viscosity sense which are of the same form as (5). For instance, let $a^{*}>0$ be such that $a(s)<a^{*}$ for any $s \geq 0$; then it can be shown that $u$ satisfies

$$
\begin{array}{r}
\min \left\{-\left[1+\frac{1-a^{*}}{a^{*}-a(|\nabla u|)}\right] \Delta_{\infty} u-\frac{|\nabla u|^{2} a(|\nabla u|)}{a^{*}-a(|\nabla u|)} \Delta u-\frac{b(|\nabla u|)}{a^{*}-a(|\nabla u|)},\right. \\
|\nabla u(x)|-\sigma\}=0,
\end{array}
$$


in the viscosity sense. If $f$ is given by (3), we can choose $a^{*}=1$ and (45) reads as

$\min \left(-\Delta_{\infty} u-|\nabla u|^{2}\left(|\nabla u|^{2}-1\right)^{+} \Delta u-|\nabla u|^{3} \sqrt{\left(|\nabla u|^{2}-1\right)^{+}},|\nabla u(x)|-1\right)=0$.

Acknowledgments. The author wishes to thank Rolando Magnanini and Simone Cecchini for the many helpful discussions. The author is grateful for the careful and thoughtful comments of the referees which led to substantial improvements over a first version of this paper.

\section{References}

[1] L. Brasco, Global $L^{\infty}$ gradient estimates for solutions to a certain degenerate elliptic equation, Nonlinear Anal., 74 (2011), pp. 516-531.

[2] L. Brasco, G. Carlier and F. Santambrogio, Congested traffic dynamics, weak flows and very degenerate elliptic equations, J. Math. Pures Appl., 93 (2010), pp. 163-182.

[3] G. Buttazzo and B. Kawohl, Overdetermined boundary value problems for the $\infty$-Laplacian, Int. Math. Res. Not. IMRN (2011), no. 2, pp. 237-247.

[4] X. Cabré and L. Caffarelli, Fully Nonlinear Elliptic Equations. Colloquium Publications 43, American Mathematical Society, Providence, RI, 1995.

[5] C. Carstensen and S. Müller, Local stress regularity in scalar nonconvex variational problems, SIAM J. Math. Anal., 34 (2002), pp. 495-509.

[6] S. Cecchini and R. Magnanini, Critical points of solutions of degenerate elliptic equations in the plane, Calc. Var. Partial Differential Equations 39 (2010), no. 1-2, pp. 121-138.

[7] G. Ciraolo, R. Magnanini and S. Sakaguchi, Symmetry of minimizers with a level surface parallel to the boundary, preprint.

[8] M.G. Crandall, H. Ishii and P.L. Lions, User's guide to viscosity solutions of second order partial differential equations, Bull. Amer. Math. Soc., 27 (1992), no. 1, pp. 1-67.

[9] G. Crasta, Existence, uniqueness and qualitative properties of minima to radially symmetric non-coercive non-convex variational problems, Math. Z., 235 (2000), pp. 569-589.

[10] I. Fragalà, F. Gazzola and B. Kawohl, Overdetermined problems with possibly degenerate ellipticity, a geometric approach, Math. Z., 254 (2006), pp. 117-132.

[11] D. Gilbarg and N.S. Trudinger, Elliptic partial differential equations of second order, Springer-Verlag, Berlin-New York, 1977.

[12] R. Jensen, Uniqueness of Lipschitz extensions: Minimizing the sup norm of the gradient, Arch. Ration. Mech. Anal., 123 (1993), pp. 51-74. 
[13] P. Juutinen, P. Lindqvist and J.J. Manfredi, The $\infty$-eigenvalue problem, Arch. Ration. Mech. Anal. 148 (1999), no. 2, pp. 89-105.

[14] S. Koike, A Beginners Guide to the Theory of Viscosity Solutions, vol. 13 of MSJ Memoirs (2004). Mathematical Society of Japan, Tokyo.

[15] R. Magnanini and G. Talenti, On complex-valued solutions to a $2 D$ eikonal equation. Part one: qualitative properties, Nonlinear Partial Differential Equations, Contemporary Mathematics 283 (1999), American Mathematical Society, pp. 203-229.

[16] R. Magnanini and G. Talenti, On complex-valued solutions to a $2 D$ eikonal equation. Part two: existence theorems, SIAM J. Math. Anal. 34 (2003), pp. 805-835.

[17] R. Magnanini and G. Talenti, On complex-valued solutions to a 2D Eikonal Equation. Part Three: analysis of a Backlund transformation, Applic. Anal. 85 (2006), no. 1-3, pp. 249-276.

[18] R. Magnanini and G. Talenti, On complex-valued 2D eikonals. Part four: continuation past a caustic, Milan Journal of Mathematics 77 (2009), no. 1, pp. 1-66.

[19] F. Santambrogio and V. Vespri, Continuity in two dimensions for a very degenerate elliptic equation, Nonlinear Anal., 73 (2010), pp. 3832-3841.

[20] P. Tolksdorf, On the Dirichlet problem for quasilinear equations in domains with conical boundary points, Comm. Partial Differential Equations, 8 (1983), pp. 773-817. 